\title{
Color Measurement and Color Theory: II. Opponent-Colors Theory
}

\author{
David H. Krantz \\ Department of Psychology, University of Michigan, \\ 330 Packard Road, Ann Arbor, Michigan 48104
}

\begin{abstract}
Quantitative opponent-colors theory is based on cancellation of redness by admixture of a standard green, of greenness by admixture of a standard red, of yellowness by blue, and of blueness by yellow. The fundamental data are therefore the equilibrium colors: the set $A_{1}$ of lights that are in red/green equilibrium and the set $A_{2}$ of lights that are in yellow/blue equilibrium. The result that a cancellation function is linearly related to the color-matching functions can be proved from more basic axioms, particularly, the closure of the set of equilibrium colors under lincar operations. Measurement analysis treats this as a representation theorem, in which the closure properties are axioms and in which the colorimetric homomorphism has the cancellation functions as two of its coordinates.

Consideration of equivalence relations based on opponent cancellation leads to a further step: analysis of equivalence relations based on direct matching of hue attributes. For additive whiteness matching, this yields a simple extension of the representation theorem, in which the third coordinate is luminance. For other attributes, precise representation theorems must await a better qualitative characterization of various nonlinear phenomena, especially the veiling of one hue attribute by another and the various hue shifts.
\end{abstract}

Part I of this series (Krantz, 1975) presented the theory of Grassmann structures. Recall that a Grassmann structure is a quadruple $\langle A, \oplus, *, \sim\rangle$, such that $\langle A, \oplus, *\rangle$ is a convex cone (Axioms 1 and 2 ), $\sim$ is an equivalence relation (Axiom 3 ), and Grassmann's additivity law holds (Axioms 4 and 5). The set $A$ is interpreted as the set of colored lights, or spectral radiance distributions; $\oplus$ and $*$ are interpreted respectively as additive color mixture and multiplication by positive scalars; and $\sim$ is the binary relation of metameric matching. Besides the relation $\sim$, which led to a trichromatic Grassmann structure (Axiom 6), we considered related measurement

This paper revises an earlier technical report with the same title, supported by NSF grants GB 4947 and GB 8181. Preparation of this manuscript was supported by NSF GB 36642X. The author is grateful to Edward Pugh for helping him to see that the need for rewriting was greater than the need for hasty publication. 
theories for dichromatic structures and for 1-chromatic Grassmann codes, such as the codes based on photopigment absorption.

From the standpoint of measurement theory, the goal of a color theory is to obtain a simultaneous homomorphic representation of as many different perceptual relations as possible. This was illustrated in part I by the nearly unique homomorphism which simultancously represents normal metameric matching and threc independent dichromatic reduction structures. In the present paper, we develop a measurement theory in which certain perceptual attributes, especially the opponent hue attributes, are simply represented.

\section{Equilibrium Colors}

\section{Achromatic Colors}

For some colors, hue is absent. We may designate these achromatic colors as a subset $B$ of the set $A$. Many classical studies involve determinations of which colors are in $B$; for example, experiments on complementary wavelengths (Sinden, 1923), least perceptible colorimetric purity (Priest \& Brickwedde, 1938), and whiteness of broad-band lights (Hurvich \& Jameson, 1951).

The metamerism relations which were the focus of part I depend only slightly, if at all, on viewing conditions (so long as the matches are symmetric, i.e., both lights are viewed under identical conditions); but perceptual qualities, such as achromaticness, vary enormously as a function of adaptation, successive contrast, and simultaneous contrast. The set $B$ should therefore be subscripted with the viewing conditions. We suppress a formal subscript only to keep notation light.

Not only the set $B$, but also the qualitative laws or axioms satisfied by $B$ may change with changes in the viewing conditions. For example, in the neutral-adapted state, with no significant contrast, it may be reasonable to expect that the additive mixture of achromatic lights is again achromatic, i.e., that $B$ is closed under the operation $\oplus$. (Curiously, this rather important property seems not to have been investigated directly.) But such an additivity property is very unlikely to hold when colors are viewed with chromatic surrounds. For example, a light $b$, which in neutral circumstances is pink, may appear achromatic in a suitably chosen red surround. Yet $b \oplus b$ (double the intensity) may again appear pink. Thus, $b$ is in $B$, yet $b \oplus b$ is not.

The term "equilibrium" is applied to achromatic colors by analogy with static equilibrium of forces. The presence of hue is analogous to the presence of acceleration, i.e., to a nonzero resultant force. The "chromatic vector" can be measured by finding a complementary color which restores achromaticity, just as the resultant force vector can be measured by equilibration. We shall see below that this is not a casual analogy: if the achromatic set $B$ is in fact additive with respect to $\oplus$, then the measurement theories of chromatic vectors and resultant force vectors (Krantz, 1973) are isomorphic. 


\section{Partial Equilibria: Opponent Hues}

Hering (1878) noted that colors can be classified as reddish or greenish or neither, but that redness and greenness are not simultaneously attributes of a color. If we add increasing amounts of a green light to a reddish light, the redness of the mixture decreases, disappears, and gives way to greenness. At the point where redness is gone and greenness is not yet present, the color may be yellowish, bluish, or achromatic. We speak of a partial chromatic equilibrium, with respect to red/green. The subset of all red/green equilibria is denoted $A_{1}$. (It goes without saying that $A_{1}$ depends on viewing conditions.)

Similarly, yellow and blue are identified as opponent hues, and we let $A_{2}$ be the subset of yellow/blue equilibrium colors. Such colors may be reddish, greenish, or achromatic, but are neither yellowish nor bluish.

Colors are in both partial equilibrium sets if and only if they are achromatic; that is,

$$
A_{1} \cap A_{2}=B
$$

Just as the chromaticness vector of a color can be evaluated in terms of the complementary, so the redness of a color can be evaluated by cancellation with a green light, and vice versa. Such quantitative evaluations were undertaken by Jameson and Hurvich (1955).

Formally, let $a_{1}$ denote a reddish stimulus and let $b_{1}$ denote a greenish one. If $c$ is not in $A_{1}$, then $c$ is either reddish, in which case there exists a positive scale factor $t$, such that $c \oplus\left(t * b_{1}\right)$ is in $A_{1}$, or else $c$ is greenish and for some $t>0, c \oplus\left(t * a_{1}\right)$ is in $A_{1}$. The cancellation coefficient $\phi_{1}(c)$ can be set equal to $t$ in the former case or to $-t$ in the latter case. If $c$ is in $A_{1}$, then nothing need be added and we set $\phi_{1}(c)=0$.

Figure 1 plots $\phi_{1}$ values measured by Jameson and Hurvich (1955) by the cancellation technique just described. For $b_{1}$, the greenish cancellation stimulus, they used a monochromatic $A_{2}$ light (490 and $500 \mathrm{~nm}$ for their two observers). For the reddish cancellation stimulus $a_{1}$, they used a $700 \mathrm{~nm}$ light, which is not an $A_{2}$ equilibrium (all the extreme long wavelength red lights appeared slightly yellowish). The unit amounts of $a_{1}, b_{1}$ are chosen so that $a_{1} \oplus b_{1}$ is an $A_{1}$ equilibrium; thus $\phi_{1}\left(a_{1}\right)=1$, $\phi_{1}\left(b_{1}\right)=-1$. The cancellation observations for Fig. 1 were obtained for a $10 \mathrm{~mL}$ equal-brightness spectrum, with a neutral white adaptation of the same brightness. The data were transformed by multiplying by luminous efficiency, so that the ordinate plotted in Fig. 1 is $\phi_{1}\left(c_{\lambda}\right)$, where $c_{\lambda}$ denotes a light of wavelength $\lambda$ and unit radiance. (For simplicity we sometimes abbreviate $\phi_{1}\left(c_{\lambda}\right)$ simply as $\phi_{1}(\lambda)$.)

Note that the endpoint of the cancellation process, for each wavelength $\lambda$, is always an $A_{1}$ equilibrium color, varying from yellowish white (for $\lambda$ in the orange or yellowgreen range) to bluish white (for $\lambda$ in the far violet part of the spectrum). The monochromatic $A_{1}$ lights appear in the yellow, around $580 \mathrm{~nm}$, and in the blue, around $475 \mathrm{~nm}$. 


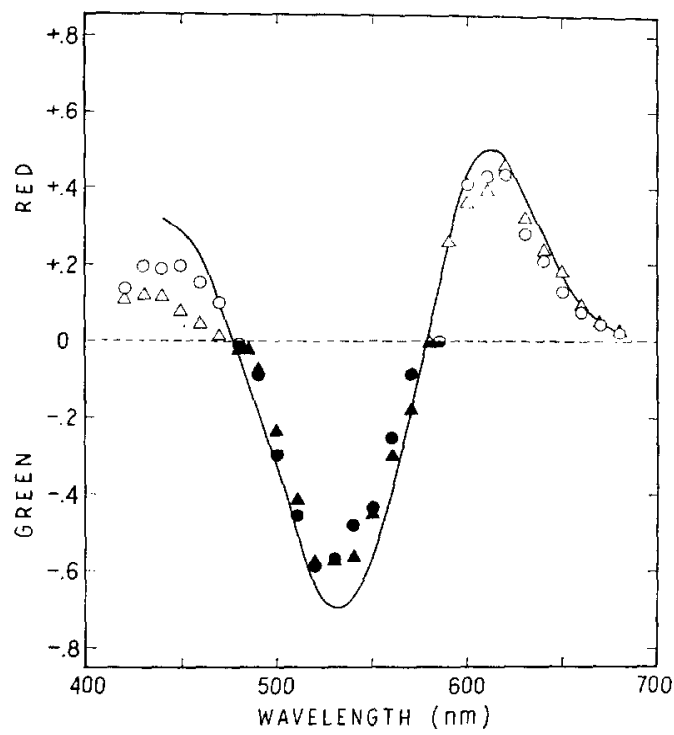

FIG. 1. Opponent-cancellation coefficients for redness (open symbols) and greenness (filled symbols) for observers $H$ (circles) and $J$ (triangles), for an equal-energy spectrum. Data replotted from Jameson and Hurvich (1955). The solid line is a linear functional for the CIE Standard Observer (Judd, 1951).

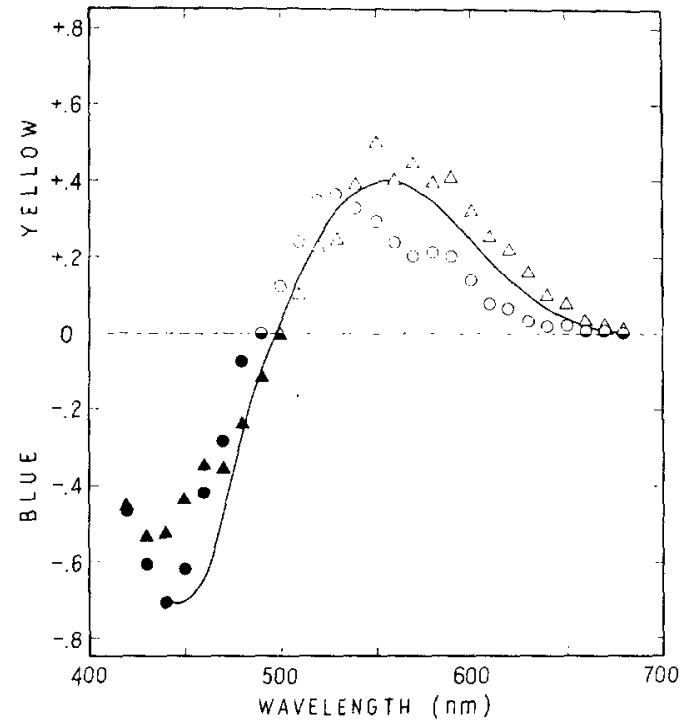

FIG. 2. Yellow/blue cancellation coefficient (details as in Fig. 1). 
Similarly, Jameson and Hurvich obtained the yellow/blue cancellation coefficient $\phi_{2}\left(c_{\lambda}\right)$, shown in Fig. 2. They employed $A_{1}$ cancellation lights: the equilibrium yellow of Fig. 1 was used to cancel blueness, in amounts shown as negative ordinates in the short-wavelength end of the spectrum, and similarly, equilibrium blue was used to obtain the $\phi_{2}$ values for long-wavelength, yellowish lights. The figure shows clearly the monochromatic $A_{2}$ equilibrium around $500 \mathrm{~nm}$ and also shows that all longwavelength lights are slightly yellowish, since a small amount of blue must be added. Other details are the same as for the red/green function of Fig. 1.

Note that measurement of a partial equilibrium is a straightforward one-dimensional procedure. The intensity of any reddish light can be varied to obtain a measure of greenness, etc. By contrast, finding a full hue equilibrium-a complement, relative to an achromatic white-is a trickier, two-dimensional operation.

\section{Linearity Assumptions in the Cancellation Procedure}

Let us try to formulate precisely the empirical facts about $A_{1}$ and $A_{2}$ equilibrium colors that must be assumed if the above-described cancellation procedure is to have a simple interpretation. First, there is the fact that cancellation is possible. This can be translated into a formal assumption as follows:

There exist $a_{1}$ and $b_{1}$ in $A$, such that for any $c$ in $A-A_{1}$, either $c \oplus\left(t * a_{1}\right)$ or $c \oplus\left(t * b_{1}\right)$ is in $A_{1}$, for some $t>0$.

The $a_{1}, b_{1}$ in question can be used as reddish and greenish cancellation stimuli. We shall not adopt this as a formal axiom, becaues it follows from other assumptions (Lemma 3 below).

Secondly, the functions of Figs. 1 and 2 would not be very meaningful if they depended on the particular choice of $a_{1}$ and $b_{1}$ used for cancellation. Changing from $a_{1}$ to $a_{1}{ }^{\prime}$ should produce a proportional change in all $\phi_{1}$ values, corresponding to a change of units. Formally we require:

If $c \oplus\left(t * a_{1}\right), d \oplus\left(u * a_{1}\right), c \oplus\left(t^{\prime} * a_{1}{ }^{\prime}\right)$, and $d \oplus\left(u^{\prime} * a_{1}{ }^{\prime}\right)$ are all in $A_{1}$, then $t / t^{\prime}=u / u^{\prime}$.

This is also a consequence of the axioms we shall assume.

Third, and most basic, the functions plotted in Figs. 1 and 2 are only useful if the cancellation coefficients $\phi_{1}$ and $\phi_{2}$ are linear functionals. That is, we require that the following equations hold:

$$
\begin{aligned}
\phi_{i}(t * a) & =t \phi_{i}(a) ; \\
\phi_{i}(a \oplus b) & =\phi_{i}(a)+\phi_{i}(b) .
\end{aligned}
$$

If Eq. (1) holds, then the cancellation coefficient is proportional to intensity. Thus, the curve for an equal-radiance spectrum allows predictions for any other radiance values. 
Without this property, it would not be useful to transform the measurements for a $10 \mathrm{~mL}$ equal-brightness spectrum to equal radiance. If in addition, Eq. (2) holds, then the curves of Figs. 1 and 2 can be used to predict the cancellation coefficients for nonspectral lights; for a light $c$ with a continuous spectral density function $c(\lambda)$ we have the integral formula $\phi_{i}(c)=\int c(\lambda) \phi_{i}(\lambda) d \lambda$.

Note that Eqs. (1) and (2) imply the independencc-of-cancellation-addend property mentioned above; for if the mentioned colors all have $\phi_{1}$ values of 0 , then we obtain $-\phi_{1}(c)=t \phi_{1}\left(a_{1}\right)=t^{\prime} \phi_{1}\left(a_{1}{ }^{\prime}\right)$ and $-\phi_{1}(d)=u \phi_{1}\left(a_{1}\right)=u^{\prime} \phi_{1}\left(a_{1}{ }^{\prime}\right)$. Therefore $t / t^{\prime}=$ $\phi_{1}\left(a_{1}^{\prime}\right) / \phi_{1}\left(a_{1}\right)=u / u^{\prime}$.

In Figs. 1 and 2 the data points are the cancellation coefficients measured for two observers, as described above, but the continuous curves are based on a particular linear functional defined on the CIE coordinates, suggested by Judd (1951). The fit is perhaps as good as can be expected, in view of the fact that there are considerable quantitative differences between the two observers, yet the linear functional is not based on the individual color-matching functions, rather on the CIE Standard Observer. Furthermore, the linear functional was not optimized to fit these data.

It might seem that the best way to test linearity would be to determine both the color-matching functions (a homomorphic representation of the Grassmann structure $\langle A, \oplus, *, \sim\rangle)$ and the cancellation function within the same ohserver and then to find the best-fitting linear relationship between them. This would be arduous. And it is unnecessary. The next two subsections show that a much simpler line of attack is decisive.

\section{Closure Properties for Equilibrium Colors}

The linearity equations (1), (2) for cancellation coefficients have two simple implications concerning the equilibrium sets $A_{i}$ : (i) closure under scalar multiplication; and (ii) closure under addition/subtraction. More precisely:

(i) If $a$ is in $A_{i}$, then $t * a$ is in $A_{i}$.

(ii) If $a$ is in $A_{i}$, then $b$ is in $A_{i}$ if and only if $a \oplus b$ is in $A_{i}$.

These properties bear a strong resemblance to Grassmann's additivity laws (Axioms 5 and 4 of Krantz, 1975) and we shall show below that in fact they lead to 1-chromatic Grassmann structures for red/green and yellow/blue cancellation equivalence. Property (i) is closely rclated to invariance of equilibrium colors with respect to the Bezold-Brücke hue shift and property (ii) is similarly related to invariance with respect to the Abney hue shift. Experimental tests of these properties, and discussion of their relations to hue-shift invariances, may be found in Larimer, Krantz, and Cicerone $(1974,1975)$.

Clearly, the truth of (i) and (ii), like the equilibrium sets $A_{1}$ and $A_{2}$ themselves, is tied to the choice of viewing conditions. It was indicated above that such closure 
properties may be expected to fail for the achromatic colors, $B=A_{1} \cap A_{2}$, under conditions of simultaneous contrast; a similar argument can be applied to each of the partial equilibrium sets, $A_{1}$ and $A_{\mathrm{2}}$.

\section{Opponent Cancellation and Metameric Matching}

Let us consider the consequences of the equilibrium-closure assumptions (i) and (ii) for metameric color matching. Let us choose as our primaries an equilibrium red $a_{1}$, an equilibrium yellow $a_{2}$, and an achromatic light $a_{3}$. That is, $a_{1}$ is a reddish $A_{2}$ light, $a_{2}$ is a yellowish $A_{1}$ light, and $a_{3}$ is in $A_{1} \cap A_{2}$. Suppose that the light to be matched, $c$, is blue-green. We can first add just enough red light to $c$ so as to cancel the greenness; i.e., we determine the cancellation coefficient, $\phi_{1}(c)=-t_{1}$, such that

$$
c \oplus\left(t_{1} * a_{1}\right)
$$

is in $A_{1}$. Next we determine the cancellation coefficient $\phi_{2}(c)=-t_{2}$, by cancelling the blueness with yellow:

$$
c \oplus\left(t_{2} * a_{2}\right)
$$

is in $A_{2}$.

Since $c \oplus\left(t_{1} * a_{1}\right)$ and $a_{2}$ are both in $A_{1}$, by the closure properties,

$$
c \oplus\left(t_{1} * a_{1}\right) \oplus\left(t_{2} * a_{2}\right)
$$

is also in $A_{1}$. But by a symmetric argument, it is also in $A_{2}$. Thereforc it is achromatic. Since $t_{3} * a_{3}$ is achromatic for every $t_{3}$ (closure property (i), for both $A_{1}$ and $A_{2}$ ), we can vary $t_{3}$ so as to produce a color match:

$$
c \oplus\left(t_{1} * a_{1}\right) \oplus\left(t_{2} * a_{2}\right) \sim t_{3} * a_{3} .
$$

Therefore the tristimulus coefficients of $c$, relative to the primaries $a_{1}, a_{2}, a_{3}$, are given by the vector

$$
\left(-t_{1},-t_{2}, t_{3}\right)=\left(\phi_{1}(c), \phi_{2}(c), t_{3}\right) .
$$

This argument shows that the cancellation coefficients $\phi_{1}$ and $\phi_{2}$ are two out of three coordinates of a homomorphic representation for $\langle A, \oplus, *, \sim\rangle$, based on a suitable choice of equilibrium colors as primaries. It follows automatically that $\phi_{\mathbf{1}}$ and $\phi_{2}$ are linear functionals on $\langle A, \oplus, *, \sim\rangle$, and can be expressed as linear combinations of any other coordinate system (see Krantz, 1975).

The above argument is really the core of this paper. (The only nonrigorous step was the assertion of a match between the two achromatic colors for suitable choice of $t_{3}$. This step is intuitive, but in the formal proof below, I shall justify it rigorously 
on the basis of trichromacy.) It shows how the closure properties (i) and (ii) lead to the conclusion that the cancellation functions $\phi_{i}$ are linear codes.

Figure 3 illustrates the above argument graphically on the CIE chromaticity diagram. The shaded triangle assumes that $b_{2}$ (equilibrium green), rather than $a_{2}$ (equilibrium red) is used as a primary, along with $a_{1}$ and $a_{3}$. (This would be more practical from the standpoint of instrumentation.) Under the closure properties (i) and (ii), the equilibrium loci $A_{1}$ and $A_{2}$ plot as straight lines in the subject's own chromaticity chart (or on the CIE one, if the subject's color matches are those of the CIE standard observer). In order for one of the color matching functions to coincide with the red/green cancellation coefficient, it must vanish on the $A_{1}$ locus, i.e., the other two of the three primaries must lie on the $A_{1}$ locus. Likewise, for another color-matching function to be the yellow/blue coefficient, two primaries must lie on the $A_{2}$ locus. Hence one primary must lie on both loci, at $a_{3}$ (white).

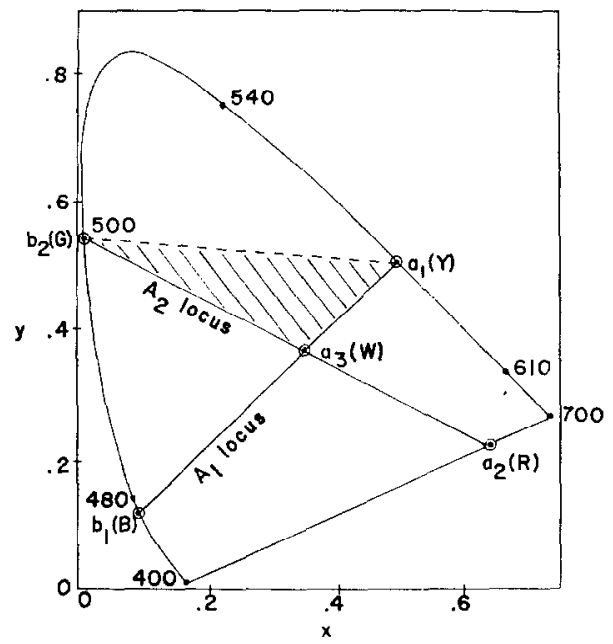

Fig. 3. CIE chromaticity diagram, showing locus of red/green equilibria (line joining equilibrium yellow $(Y)$ to equilibrium blue $(B)$ ) and locus of yellow/blue equilibria (line joining equilibrium red $(R)$ to equilibrium green $(G)$ ). This plot assumes that both equilibrium loci satisfy the Grassmann-like closure properties (i) and (ii). The shaded triangle is the set of lights metameric to a positive mixture of equilibrium yellow, green, and white $(W)$ primaries. For spectral lights between $B$ and $G$, a negative amount of primary $Y$ is needed to match and the final match lies on the $A_{2}$ locus. For spectral lights between $700 \mathrm{~nm}$ and $Y$, a negative amount of primary $G$ is needed and the final match lies on the $A_{1}$ locus. For spectral lights between $G$ and $Y$, the match consists of a mixture of those two primaries, with a negative amount of the $W$ primary; the match locus is on the line joining $G$ and $Y$. Spectral lights between $400 \mathrm{~nm}$ and $B$ require negative amounts of both the $G$ and the $Y$ primaries; the redness and blueness are canceled and the final match locus lies at $W$. The same holds for lights that are mixtures of $400 \mathrm{~nm}$ and $700 \mathrm{~nm}$, if they lie between $400 \mathrm{~nm}$ and the equilibrium red point $R$. See text for further comments. 
Representation and Uniqueness Theorem for Opponent-Colors Theory

The results of the previous section suggest that the following representation theorem can be proved: given a structure $\left\langle A, \oplus, *, \sim, A_{1}, A_{2}\right\rangle$, we can find a homomorphism $\phi$ into a vector structure $\left\langle V,+, \cdot,=, V_{1}, V_{2}\right\rangle$, where $V$ is a threedimensional vector space and $V_{1}, V_{2}$ are subspaces defined by $\phi_{1}=0$ and $\phi_{2}=0$, respectively. The axioms needed are Axioms (1)-(6) of a trichromatic Grassmann structure (Krantz, 1975); Axiom (7), which links metameric matching to equilibria by asserting that metameric lights are in identical equilibrium sets; Axiom (8), embodying the equilibrium-closure properties (i) and (ii) discussed above; and an Axiom (9), which asserts the existence of suitable cancellation lights. The numbering of axioms, theorems, and lemmas is continued from Krantz (1975).

THEOREM 6. Let $\langle A, \Theta, *, \sim\rangle$ be a trichromatic proper Grassmann structure and let $A_{1}, A_{2}$ be subsets of $A$ such that for $a, b$ in $A$ :

(7) if $a \sim b$ and $a$ is in $A_{i}\left(i=1\right.$ or 2), then $b$ is in $A_{i}$;

(8) (i) if $a$ is in $A_{i}$ and $t>0$, then $t * a$ is in $A_{i}$;

(ii) if $a$ is in $A_{i}$, then $b$ is in $A_{i}$ if and only if $a \oplus b$ is in $A_{i}$;

(9) there exist $a_{1}, b_{1}$ in $A_{2}$ but not in $A_{1}$, such that $a_{1} \oplus b_{1}$ is in $A_{1}$, and there exist $a_{2}, b_{2}$ in $A_{1}$ but not in $A_{2}$, such that $a_{2} \oplus b_{2}$ is in $A_{2}$.

Then there exist real-valued functions $\phi_{1}, \phi_{2}, \phi_{3}$ on $A$ satisfying the following properties:

(i) $\phi=\left(\phi_{1}, \phi_{2}, \phi_{3}\right)$ is a homomorphism of $\left\langle A, \oplus, *, \sim\right.$ into $\left\langle\mathrm{Re}^{3},+, \cdot, \Rightarrow\right\rangle$, satisfying (i)-(iv) of Theorem 1 ;

(ii) for $i=1,2 a$ is in $A_{i}$ if and only if $\phi_{i}(a)=0$.

Moreover if $\phi_{1}{ }^{\prime}, \phi_{2}{ }^{\prime}, \phi_{3}{ }^{\prime}$, are any other functions having properties (i) and (ii), then there are constants $\alpha_{1}, \alpha_{2}, \beta_{1}, \beta_{2}$, and $\beta_{3}$ such that $\alpha_{1}, \alpha_{2}$ and $\beta_{3} \neq 0$ and

$$
\left(\begin{array}{l}
\phi_{1}^{\prime} \\
\phi_{2}^{\prime} \\
\phi_{3}^{\prime}
\end{array}\right)=\left(\begin{array}{lll}
\alpha_{1} & 0 & 0 \\
0 & \alpha_{2} & 0 \\
\beta_{1} & \beta_{2} & \beta_{3}
\end{array}\right)\left(\begin{array}{c}
\phi_{1} \\
\phi_{2} \\
\phi_{3}
\end{array}\right) .
$$

That is, $\phi_{1}$ and $\phi_{2}$ are unique up to changes of unit and \pm orientation.

It will be shown in the proof that the functions $\phi_{1}$ and $\phi_{2}$, which are unique except for choice of unit and sign, are determined by cancellation.

Axiom (7) asserts only that if light $a$ is an equilibrium light and $b$ is not, then $a \sim b$ is false. In conjunction with the closure axiom, it shows that the cancellation coefficient is a code (Krantz, 1975) for the Grassmann structure $\langle A, \Theta, *, \sim\rangle$. Axiom (8) was discussed above. Note that both these axioms are consequences of the theorem's conclusion; they are logically necessary for the representation. 
Axiom (9) postulates the existence of two equilibrium red/greens, $a_{1}, b_{1}$, whose additive mixture is neither reddish nor greenish (therefore $a_{1} \oplus b_{1}$ is achromatic, by Axiom 8 ). Similarly, $a_{2}, b_{2}$ are complementary equilibrium yellow and blue. Cancellation coefficients can of course be measured without using equilibrium colors as cancellation stimuli, and indeed, it is convenient to use a monochromatic nonequilibrium red, as Jameson and Hurvich did. The present assumption could be replaced by one which says in effect that cancellation is always possible, and indeed, this will be sketched below in order to obtain a theorem valid for the situation in which only one of the sets $A_{i}$ satisfies closure. The reason for keeping Axiom (9) in its present form is that the use of equilibrium cancellation stimuli allows us to obtain the relationship between cancellation cocfficients and metameric matching directly, as sketched at the end of the preceding section.

Axiom (9) also defines the units of $b_{i}$ relative to $a_{i}$ by specifying that $a_{i}, b_{i}$ cancel one another. This saves introducing correction factors.

Finally, note that we retain from the theorems of Part I the assumption that the Grassmann structure is proper, i.e., no equation of form $a \oplus b \sim a$ can hold.

Proof of Theorem 6. Let $a_{1}, a_{2}, b_{1}, b_{2}$ be elements of $A$ with the properties postulated in Axiom (9). Let $a_{3}$ be an arbitrary element of $B$, e.g., $a_{3}=a_{1} \oplus b_{1}$. In Lemmas 2 and 3, we show that $a_{1}, a_{2}, a_{3}$ are a set of primaries (see Axiom (6ii) in part I) and that $a_{i}, b_{i}$ can be used for cancellation, $i=1,2$.

LeMma 2. $a_{1}, a_{2}, a_{3}$ are a set of primaries.

Proof. Supposc not; then there is a color equation

$$
\sum_{i=1}^{3} t_{i} * a_{i} \sim \sum_{i=1}^{3} u_{i} * a_{i},
$$

with $t_{i} \neq u_{i}$ for at least one $i$. Now add

$$
\left(u_{1} * b_{1}\right) \oplus\left(u_{2} * b_{2}\right)
$$

to both sides of (4). By additivity of color matches (Axiom 4), and rearranging terms on each side (Axioms 1-3), we obtain

$$
\begin{array}{r}
\left(t_{1} * a_{1}\right) \oplus\left(u_{1} * b_{1}\right) \oplus\left(t_{2} * a_{2}\right) \oplus\left(u_{2} * b_{2}\right) \oplus\left(t_{3} * a_{3}\right) \\
\sim\left(u_{1} *\left(a_{1} \oplus b_{1}\right)\right) \oplus\left(u_{2} *\left(a_{2} \oplus b_{2}\right)\right) \oplus u_{3} * a_{3} .
\end{array}
$$

By Axiom (8), the right side of (5) is in $B$, hence by Axiom (7), so is the left side. Applying Axiom (8) to the left side we obtain that

$$
\begin{aligned}
& \left(t_{1} * a_{1}\right) \oplus\left(u_{1} * b_{1}\right) \text { is in } A_{1} ; \\
& \left(t_{2} * a_{2}\right) \oplus\left(u_{2} * b_{2}\right) \text { is in } A_{2} .
\end{aligned}
$$


Using Axiom (8) again we infer that $t_{1}=u_{1}$ and $t_{2}=u_{2}$ (e.g., if $t_{1}>u_{1}$, then $\left(\left(t_{1}-u_{1}\right) * a_{1}\right) \oplus\left(u_{1} *\left(a_{1} \oplus b_{1}\right)\right.$ is in $A_{1}$, so $a_{1}$ is in $A_{1}$, contrary to fact $)$. But by subtractivity (Axiom 4), Eq. (4) reduces to $t_{3} * a_{3} \sim u_{3} * a_{3}$, with $t_{3} \neq u_{3}$; this is impossible in a proper Grassmann structure (e.g., if $t_{3}>u_{3}$, then $\left(\left(t_{3}-u_{3}\right) * a_{3}\right) \oplus$ $\left.\left(u_{3} * a_{3}\right) \sim u_{3} * a_{3}\right)$. We conclude that Eq. (4) cannot hold nontrivially, i.e., if it holds, then $t_{i}=u_{i}, i=1,2,3$.

Q.E.D.

An intuitive restatement of the proof of Lemma 2 may be helpful. If a color equation (4) obtains, involving equilibrium red, yellow, and white, then we can cancel the redness and yellowness on the right side of the equation by adding appropriate amounts of equilibrium green and blue to both sides. Since the right side is achromatic, so is the left; hence the coefficients of the red and yellow components must have been equal in the original equation. Eliminating them from the equation gives an achromatic color equation, which is then also trivial (isomeric). So the original equation must have been trivial.

Lemma 3. For $i=1,2$ and for any $c$, either $c$ is in $A_{1}$ or there exists a unique $t_{i}>0$ such that either $c \oplus\left(t_{i} * a_{i}\right)$ or $c \oplus\left(t_{i} * b_{i}\right)$ is in $A_{i}$. That is, $a_{i}, b_{i}$ can be used to determine a unique cancellation coefficient for any $c$.

Proof. By trichromacy (Axiom 6i) there is a nontrivial color equation involving $c, a_{1}, a_{2}$, and $a_{3}$. The coefficients of $c$ on the two sides must be unequal (otherwise it could be reduced to a nontrivial equation in the $a_{i}$, contradicting Lemma 2). By Axioms (1)-(5) we can write the equation as

$$
c \oplus \sum_{j=1}^{3}\left(s_{j} * a_{j}\right) \sim \sum_{j=1}^{3}\left(u_{j} * a_{j}\right) .
$$

Now add $u_{i} * b_{i}$ to both sides of (6) $(i=1$ or 2$)$. By Axiom (8) the right side of the new equation is in $A_{i}$, therefore by Axiom (7) so is the left side; by Axiom (8) again,

$$
c \oplus\left(s_{i} * a_{i}\right) \oplus\left(u_{i} * b_{i}\right)
$$

is in $A_{i}$. So if $s_{i}>u_{i}$, let $t_{i}=s_{i}-u_{i}$ and obtain $c \oplus\left(t_{i} * a_{i}\right)$ in $A_{i}$; or if $u_{i}>s_{i}$ let $t_{i}=u_{i}-s_{i}$ and obtain $c \oplus\left(t_{i} * b_{i}\right)$ in $A_{i}$; or if $s_{i}=u_{i}$, then $c$ is in $A_{i}$.

To show the uniqueness of the cancellation coefficient we examine the various possiblc cascs. For example if $c \oplus\left(t_{i} * a_{i}\right)$ and $c \oplus\left(t_{i}{ }^{\prime} * a_{i}\right)$ are both in $A_{i}$, with $t_{i}-t_{i}^{\prime}>0$, we have the identity

$$
c \oplus\left(t_{i}^{\prime} * a_{i}\right) \oplus\left(\left(t_{i}-t_{i}^{\prime}\right) *\left(a_{i} \oplus b_{i}\right)\right)=c \oplus\left(t_{i} * a_{i}\right) \oplus\left(\left(t_{i}-t_{i}^{\prime}\right) * b_{i}\right) .
$$

However by Axiom (8) the left side is in $A_{i}$ and the right side is not, which is impossible Similarly if $c \oplus\left(t_{i} * a_{i}\right)$ and $c \oplus\left(t_{i}^{\prime} * b_{i}\right)$ are both in $A_{i}$, we have the identity

$$
c \oplus\left(t_{i} * a_{i}\right) \oplus\left(t_{i}^{\prime} *\left(a_{i} \oplus b_{i}\right)\right)=c \oplus\left(t_{i}^{\prime} * b_{i}\right) \oplus\left(\left(t_{i}+t_{i}{ }^{\prime}\right) * a_{i}\right) .
$$


Again the left side is in $A_{i}$ but the right is not. The other two cases are handled similarly. Thus $t_{i}$ is unique.

Q.E.D.

An intuitive proof of Lemma 3 is very simple. If red, yellow, and white are used to match $c$, then if a net amount of red is added to $c$ in the match, that amount defines the cancellation coefficient; while if a net amount of red occurs on the right side of Eq. (6), it can be cancelled by equilibrium green, and that amount of green defines the cancellation coefficient.

It is now easy to complete the proof of Theorem 6 . Let $\phi$ be a homomorphism of $\langle A, \oplus, *, \sim\rangle$ into $\langle V,+, \cdot, \Rightarrow$ satisfying properties (i)-(iv) of Theorem 1. Let $\phi_{1}(c), \phi_{2}(c), \phi_{3}(c)$ be the coordinates of $\phi(c)$ relative to the primaries $a_{1}, a_{2}, a_{3}$, i.e., relative to the basis $\phi\left(a_{1}\right), \phi\left(a_{2}\right), \phi\left(a_{3}\right)$ in $V$. (Lemma 1 and Theorem 3 assure that the $\phi\left(a_{i}\right)$ are independent.) By definition, if Eq. (6) holds, then

$$
\phi_{i}(c)=u_{i}-s_{i}, \quad i=1,2,3 .
$$

By the proof of Lemma $3, \phi_{i}(c)=0$ if and only if $c$ is in $A_{i} ; \phi_{i}(c)$ is precisely the cancellation coefficient relative to $A_{i}$.

To prove the uniqueness statement, let $\phi_{1}{ }^{\prime}, \phi_{2}{ }^{\prime}, \phi_{3}{ }^{\prime}$ be any other functions with the same properties. For $i=1,2$, let $\alpha_{i}=\phi_{i}{ }^{\prime}\left(a_{i}\right)$. Since $a_{i}$ is not in $A_{i}, \alpha_{i} \neq 0$. Since $a_{i} \oplus b_{i}$ is in $A_{i}$ we have $\phi_{i}^{\prime}\left(b_{i}\right)=-\alpha_{i}$.

Suppose that $\phi_{i}(c)>0$. Then we have from Lemma 3 that $c \oplus\left(\phi_{i}(c) * b_{i}\right)$ is in $A_{i}$; hence

$$
\begin{aligned}
\phi_{i}{ }^{\prime}\left(c \oplus\left(\phi_{i}(c) * b_{i}\right)\right) & =0 \\
& =\phi_{i}{ }^{\prime}(c)+\phi_{i}(c) \phi_{i}{ }^{\prime}\left(b_{i}\right) \\
& =\phi_{i}{ }^{\prime}(c)-\alpha_{i} \phi_{i}(c) .
\end{aligned}
$$

Similarly, if $\phi_{i}(c)<0$ we have $\phi_{i}{ }^{\prime}(c)=\alpha_{i} \phi_{i}(c)$. This completes the proof of the theorem.

\section{1-Chromatic Grassmann Structures Based on Equilibrium Sets}

In this section we develop a slightly different approach to the representation and uniqueness theorem for opponent-colors theory. We define $a \sim_{i} b$ if and only if $a$ and $b$ are cancelled by the same stimulus relative to $A_{i}$, i.e., if and only if $a \oplus c$ and $b \oplus c$ are in $A_{i}$ for some $c$. Under our assumptions, $\left\langle A, \oplus, *, \sim_{i}\right\rangle$ is an (improper) 1-chromatic Grassmann structure and in fact is a Grassmann code for the trichromatic structure $\langle A, \oplus, *, \sim\rangle$, corresponding to the lincar code $\phi_{i}$.

The importance of this alternative approach is threefold. First, it ties in the properties of equilibria more precisely with those of Grassmann codes, discussed in part I. Second, it provides a linear representation for either $A_{1}$ or $A_{2}$ to cover cases where the basic closure axiom (Axiom 8 ) is satisfied by oniy one of the equilibrium 
sets, rather than both as required by Theorem 6 . 'I'hird, it raises the question of other Grassmann or nonGrassmann codes defined by perceptual equivalence relations; that topic will become the dominant focus of the remainder of this paper.

Lemma 4. Let $\langle A, \oplus, *\rangle$ satisfy Axioms (1) and (2) and let $A_{i}$ be a subset of $A$ satisfying closure (Axiom 8). Suppose that for any $a, b$ in $A$, either

(i) for some $t>0, a \oplus(t * b)$ is in $A_{i}$, or

(ii) for some $t>0$ and $c$ in $A$, both $a \oplus c$ and $(t * b) \oplus c$ are in $A_{i}$. Define $a \sim_{i} b$ if there exists $c$ such that $a \oplus c, b \oplus c$ are in $A_{i}$. Then $\left\langle A, \oplus, *, \sim_{i}\right\rangle$ is $a$ 1-chromatic improper Grassmann structure.

In other words, if $A_{i}$ is an equilibrium subset of lights which is closed under addition and scalar multiplication, such that any two elements of $A$ either cancel or are canceled by a common light $c$ (after adjustment of relative intensity), then cancellation equivalence defines a 1-chromatic Grassmann structure.

Proof. First we show $\sim_{i}$ is an equivalence relation (Axiom (3) of Grassmann structures). Symmetry (3ii) is obvious. For reflexivity, note that if $a$ is in $A_{i}$, then $a \oplus a$ is in $A_{i}$, whence $a \sim_{i} a$; or if $a$ is not in $A_{i}$, then $a \oplus(t * a)$ is never in $A_{i}$, so by (ii) of the hypotheses we have $a \oplus c$ in $A_{i}$, whence $a \sim_{i} a$. For transitivity, note that if $a \oplus c, a^{\prime} \oplus c, a^{\prime} \oplus d$, and $a^{\prime \prime} \oplus d$ are all in $A_{i}$, then $a \oplus\left(a^{\prime} \oplus c \oplus d\right)$ and $a^{\prime \prime} \oplus\left(a^{\prime} \oplus c \oplus d\right)$ are in $A_{i}$, whence $a \sim_{i} a^{\prime \prime}$.

Secondly we show additivity (Axiom 4). Suppose $a \sim_{i} a^{\prime}$. Then we can find $c$ with $a \oplus c, a^{\prime} \oplus c$ in $A_{i}$, and for any $b$ we can find $d$ with $b \oplus d$ in $A_{i}$ (reflexivity of $\left.\sim_{i}\right) ;(a \oplus b) \oplus(c \oplus d)$ and $\left(a^{\prime} \oplus b\right) \oplus(c \oplus d)$ are in $A_{i}$, so $a \oplus b \sim_{i} a^{\prime} \oplus b$. The converse (subtractivity) is immediate. So is the scalar multiplication law. So $\left\langle A, \oplus, *, \sim_{i}\right\rangle$ is a Grassmann structure. It is clearly improper since $a \oplus b \sim_{i} a$ if $b$ is in $A_{i}$.

Finally, for 1-chromacy, it is easiest to construct the vector representation $\phi$ (Theorem 1) and to note that the hypothesis guarantees either $\phi(a)=-t \cdot \phi(b)$ or $\phi(a)=-\phi(c)=t \cdot \phi(b)$, so any two vectors are linearly dependent.

Q.E.D.

Theorem 7. Let the hypotheses of Lemma 4 hold, and in addition, let $\langle A, \oplus, *, \sim\rangle$ be a Grassmann structure and let Axiom (7) hold. Then $\sim_{i}$ includes $\sim$; so that any representation for $\left\langle A, \Theta, *, \sim_{i}\right\rangle$ is a linear code for $\langle A,(\oplus, *, \sim\rangle$. (See part 1.)

The proof is easy and will not be given formally. It amounts to saying that if $a, b$ are metameric and $c$ cancels $a$, then since $a \oplus b, b \oplus c$ are also metameric, $c$ cancels $b$, and $a, b$ are thus cancellation equivalent.

Lemma 4 and Theorem 7 show clearly the construction of a linear code for a Grassmann structure from an equilibrium set satisfying Axioms (7) and (8). They 
thus deal with the case where only a single equilibrium set satisfies the crucial closure axioms, (8i) and (8ii). (See Larimer, Krantz, and Cicerone, 1975) for evidence showing a small nonadditivity in the yellow/blue equilibrium set.) The 1-chromacy of the cancellation structure is guaranteed by the main auxiliary hypothesis of Lemma 4 , that any two lights either cancel or are canceled by a common light. If both of the cancellation structures satisfy Axioms (7) and (8), however, this auxiliary hypothesis can be deduced using Axiom (9) (see Lemma 3).

Referring to Fig. 3, we envisage a situation in which $A_{1}$ plots as a straight line in the chromaticity chart, but where $A_{2}$ is curved or perhaps cannot even be plotted on the chromaticity chart (if Axiom (8i) fails). One can still choose two primaries on the $A_{1}$ locus and the third primary anywhere off that locus; the color-matching function for the third primary will be the red/green cancellation coefficient.

\section{The Structure of Perceptual Attributes}

In this section we move away from the study of equilibrium colors, which was the focus of the early part of the paper, and consider perceptual qualities of nonequilibrium colors. The same sequence was followed by Hurvich and Jameson. Their first paper (Jameson \& Hurvich, 1955) dealt principally with measurement of chromatic responses by cancellation; their second one (Hurvich \& Jameson, 1955) took up the relation of the cancellation codes to the classical attributes of perceived color: brightness, saturation, and hue. My treatment of these latter questions, however, will be far less ambitious than theirs. Broadly speaking, I will confine myself almost entirely to phenomena of heterochromatic and heteroluminous matching relations, and will not, in this paper, venture into the other vast areas of color-perception data: color discrimination, adaptation and contrast, and color defects.

\section{A Nonlinear Model of Red/Green Perception}

To avoid excessive abstractness, I will introduce the various problems and concepts by reference to a specific model of the code underlying perceived redness and greenness. I don't think that there is much chance that this particular model is correct; its only virtues are that it is not obviously incorrect, and it is therefore complicated enough to illustrate a number of important points.

Let us denote by $\alpha, \beta$, and $\gamma$ the linear codes corresponding to the sensitivity curves of the short-wavelength, middle-wavelength, and long-wavelength cone pigments, respectively. Let $f_{1}$ denote a quantitative measure of perceived redness/greenness. The model consists of the following relation between $f_{1}$ and $\alpha, \beta, \gamma$ :

$$
f_{1}=h\left(w_{3} \gamma-w_{2} \beta\right)-h\left(w_{1} \beta-\alpha\right) .
$$


In effect, three cancellation or subtractive processes are involved. There is an opposition between long-wavelength and middle-wavelength cones, $\gamma, \beta$, resulting in a linear signal $w_{3} \gamma-w_{2} \beta$; and an opposition between middle- and short-wavelength cones, producing the linear signal $w_{1} \beta-\alpha$ (we choose units such that the coefficient of $\alpha$ is unity). These two linear signals go through a compressive transformation, described by the strictly increasing function $h$. (For example we may try $h(x)=$ $p x /\left(p^{2}+x^{2}\right)^{1 / 2}$, which is symmetrically compressive for positive and negative values of the input signal $x$. The parameter $p$ determines the level at which $h$ becomes substantially nonlinear.) The two compressed outputs then interact subtractively to yield the net signal $f_{1}$. Positive values of $f_{1}$ correspond to perceived redness, negative ones to perceived greenness.

If $a$ is a red/green equilibrium color, then $f_{1}(a)=0$. From Eq. (8), this means that $h\left(w_{3} \gamma(a)-w_{2} \beta(a)\right)=h\left(w_{1} \beta(a)-\alpha(a)\right)$; and since $h$ is the same for both signals, we conclude that $\phi_{1}(a)=0$, where

$$
\phi_{1}(a)=w_{3} \gamma(a)-\left(w_{2}+w_{1}\right) \beta(a)+\alpha(a) .
$$

This shows that the present model is consistent with additive closure of $A_{1}$, and that the corresponding linear code $\phi_{1}$ is related to the cone pigments by Eq. (9). Yet $f_{1}$ is nonlinear and is not related in any simple or one-to-one way to $\phi_{1}$.

\section{Codes Based on Matching}

Previously, we have considered two equivalence relations, $\sim_{1}$ and $\sim_{2}$, based on $\mathrm{red} /$ green and yellow/blue equilibria, and we showed that additivity of the equilibrium set implies additivity of the corresponding cancellation-equivalence relation. In this case, $\sim_{i}$ is represented by equal values of a linear code $\phi_{i}$; this linear code can be written as a linear combination of any linear coordinates and in particular, it can be expressed as a linear combination of cone-pigment codes, as in (9). But suppose that reddish lights $a, b$ satisfy $a \sim_{1} b$; i.e., $a, b$ are equivalent in redness, via the indirect cancellation test. What about a direct perceptual comparison? Do they look equally red? More generally, we can consider alternative equivalence relations, $\sim_{1}$ ' and $\sim_{2}{ }^{\prime}$, based on direct heterochromatic and heteroluminous comparisons of redness/greenness and of yellowness/blueness. Does $\sim_{i}^{\prime}$ coincide with $\sim_{i}$ ? If not, what are the properties of $\sim_{i}{ }^{\prime}$; for example, is $\left\langle A, \oplus, *, \sim_{i}{ }^{\prime}\right\rangle$ a Grassmann structure ? The model embodied in Eq. (8) shows one way in which $\sim_{1}$ and $\sim_{1}^{\prime}$ could fail to coincide. The functions $f_{1}$ and $\phi_{1}$ both relate to the photopigments, and they are zero together, on $A_{1}$, positive together for reddish lights, and negative together for greenish lights. But $f_{1}$ cannot be written as a simple function of $\phi_{1}$ alone. For example if $\phi_{1}(b)=0$, then $\phi_{1}(a \oplus b)=\phi_{1}(a)$; yet $f_{1}(a \oplus b)$ will be unequal to $f_{1}(a)$, if $h$ is nonlinear. Since $\sim_{1}^{\prime}$ corresponds to $f_{1}$-equality and $\sim_{1}$ corresponds to $\phi_{1}$-equality, it follows that $\sim_{1}{ }^{\prime}$ and $\sim_{1}$ do not coincide in this model. (Nor does $\sim_{1}{ }^{\prime}$ satisfy 
additivity or scalar multiplication axioms, except for special forms of $h$; so $f_{1}$ may be neither a Grassmann nor an invariant code: see part I.)

Redness/greenness and yellowness/blueness are not the only attributes that can be matched, of course. The classical perceptual qualities of brightness, saturation, and hue can all be compared directly across stimuli. Though all these sorts of judgments are quite difficult, they do yield reasonably consistent data. Heterochromatic matching of brightness has been investigated quite extensively; heteroluminous matching of hue has been used in investigations of the Bezold-Brücke hue shift; and both heterochromatic and heteroluminous matching of saturation produced excellent results in one study by Indow and Stevens (1966). Corresponding to these attributes we may postulate equivalence relations $\sim_{B}, \sim_{S}$, and $\sim_{H}$.

In addition, we need to ask about the third opponent attribute of the Hering theory: white/black. Why is there no equilibrium structure? Can a third Grassmann code be obtained by direct heterochromatic whiteness matching?

The goal of theory is to determine the structural equations that interrelate these various attributes. The model embodied in Eqs. (8) and (9) illustrates one part of a proposed solution; Eq. (8) relates $f_{1}$, the perceived red/green, to cone pigments and thus indirectly to cancellation coefficients. Even if we knew the function $f_{1}$, as well as $f_{2}$ for perceived yellow/blue, we would still need to know how these relate to hue matching, $\sim_{H}$. For example, does the Hurvich-Jameson hue coefficient $f_{1} /\left(f_{1}+f_{2}\right)$ predict hue matches?

The measurement theory approach to these questions is based on the correspondence between qualitative laws (or axioms) and quantitative descriptions. Given a proposed function such as (8), we must ask what axioms characterize it. Or given the laws of the phenomenon under study (hue matching, for example) we must ask what functions satisfy the axioms. In many cases, these questions may be premature; much more needs to be learned about the phenomenon before the functions describing it can be guessed.

In the remainder of this paper, I shall briefly survey the status of matching relations for opponent attributes, brightness, hue, and saturation from the above standpoint.

\section{Opponent Hue Attributes and the Veiling Phenomenon}

We have already noted that direct matching of a hue attribute yields an equivalence relation $\sim_{i}{ }^{\prime}$, which does not coincide with the cancellation equivalence relation, $\sim_{i}$. If $b$ is in $A_{i}$, then for any $a, a \oplus b \sim_{i} a$. But we cannot expect that $a \oplus b \sim_{i}^{\prime} a$. For example, suppose that $a$ is an equilibrium green and $b$ is an equilibrium blue. The mixture $a \oplus b$ will be less greenish than $a$ was. In the limit, if the blue is sufficiently intense, the greenness all but disappears.

I shall use the term veiling to describe this phenomenon. It seems to have been neglected in the color literature. 
Hurvich and Jameson (1951) reported that broad-band lights, chromatic at low luminances, are judged "white" if the luminance is raised sufficiently. We may interpret this as a fact about veiling: whiteness veils hue attributes, and to an increasing extent at high luminance.

If we could adopt as an axiom the equality of the relations $\sim_{i}^{\prime}$ and $\sim_{i}$, this would imply a simple quantitative relation between the cancellation code $\phi_{i}$ and the perceived hue magnitude $f_{i}$. They would be linked by a function of one variable:

$$
f_{i}=f_{i}\left(\phi_{i}\right) \text {. }
$$

Because of the veiling phenomenon, this axiom and the resulting equation must be rejected; $f_{i}$ must depend on at least one other code besides $\phi_{i}$.

Since $\sim_{i}^{\prime}$ must be considered as a distinct entity, the next question is might $\left\langle A, \oplus, *, \sim_{i}^{\prime}\right\rangle$ be a Grassmann structure ? The following plausible facts are sufficient to reject this possibility. Let $a$ be a bright green light and $b$ a blue light so intense that $a \oplus b$ is only slightly greenish (veiling). Let $c$ be a greenish yellow, at a much lower luminance, selected such that $a \oplus b \sim_{1}^{\prime} c$ (match in perceived greenness). Now add a red light $d$ to both sides, such that $a \oplus d$ is in red/green equilibrium. Then $\boldsymbol{a} \oplus \boldsymbol{b} \oplus d$ will be a red/green equilibrium, but $c \oplus \boldsymbol{d}$ will be reddish. Thus, $a \oplus b \oplus d \sim_{1}{ }^{\prime} c \oplus d$ fails.

The above results show that measurement axioms can serve as useful guides to thinking, even in cases when they are rejected. We conclude that $\sim_{i}^{\prime} \neq \sim_{i}$ and that $\sim_{i}{ }^{\prime}$ is nonadditive. We do not yet know what laws it does satisfy; so, much work remains before we can hope to pin down the structural relations of these hue attributes to other codes.

Note that the red/green model presented in Eq. (8) is capable of predicting veiling and also predicts the nonadditivity of $\sim_{1}{ }^{\prime}$. Specifically, let $q(a)=w_{3} \gamma(a)-w_{2} \beta(a)$ and $r(a)=w_{1} \beta(a)-\alpha(a)$. If we add an equilibrium light $b$, with $q(b)=r(b)=w$, we have from (8)

$$
f_{1}(a \oplus b)=h(w+q(a))-h(w+r(a)) .
$$

For suitably chosen nonlinear $h$ (the function suggested above, $h(x)=p x /\left(p^{2}+x^{2}\right)^{1 / 2}$, will do), $f_{1}$ approaches 0 as $w$ approaches $\pm \infty$. This shows that veiling can occur. And moreover, since $f_{1}$ cannot be written as a function of a single linear combination of $\alpha, \beta, \gamma$, it is not a Grassmann code.

Note that in this model, the veiling by equilibrium lights is a function of a single linear code, denoted above by $q(b)$. This implies that veiling effects satisfy restricted additivity properties. ${ }^{1}$ These have not yet been tested experimentally, nor is it known yet precisely what class of models (including Eq. (8)) predicts such properties.

In short, the functional description of the opponent hue attributes is closely tied

1 This was pointed out to the author by James R. Moeller. 
to the empirical properties of the veiling phenomenon. This is a problem area in which little is known.

\section{Brightness and Luminance Codes}

In this section we formulate a more fine-grained representation theorem for opponent colors, in which the first two coordinates, $\phi_{1}$ and $\phi_{2}$, are the cancellation codes for red/green and yellow/blue, and the third coordinate, $\phi_{3}$, is related to luminance or to whiteness.

Hurvich and Jameson could not measure whiteness/blackness by cancellation, because blackness depends on contrast. In the standard form of their representation, they used for their third coordinate the $Y$-coefficient, or luminance, in the $1931 \mathrm{CIE}$ $X, Y, Z$ system. This is by definition a linear functional, which is closely related to the results of certain matching relations.

Let us assume the existence of a heterochromatic "whiteness" matching relation $\sim_{3}$ which yields a 1-chromatic Grassmann structure. The exact nature of this relation will be discussed briefly later; clearly it cannot be brightness matching, since that is known to violate additivity. Specifically, we supplement our previous Axioms (1)-(9) with the following:

(7') If $a \sim b$, then $a \sim 3$.

(10) $\left\langle A, \oplus, *, \sim_{3}\right\rangle$ is a proper 1-chromatic Grassmann structure.

Axiom $\left(7^{\prime}\right)$ is closely related to Axiom (7) for equilibrium sets: metameric lights have identical appearance and so they match in whiteness. Axiom (10) includes a good many separate assertions (Axioms (3)-(6) of Grassmann structures, with their various subparts) but the main empirically vulnerable part is Abney's Law, or Grassmann's fourth law: $a \sim_{3} b$ if and only if $a \oplus c \sim_{3} b \oplus c$.

Theorem 8. Let $\left\langle A, \oplus, *, \sim, A_{1}, A_{2}, \sim_{3}\right\rangle$ satisfy Axioms (1)-(6), (7), ( $\left.7^{\prime}\right)$, and (8)-(10). Let $\sim_{1}, \sim_{2}$ be defined as in Lemma 4. There exist real-valued functions $\phi_{1}, \phi_{2}$, $\phi_{3}$ on $A$ such that

(i) $\phi=\left(\phi_{1}, \phi_{2}, \phi_{3}\right)$ is a vector representation of $\langle A, \oplus, *, \sim\rangle$, satisfying (i)-(iv) of Theorem 1;

(ii) for $i=1,2,3, \phi_{i}$ is a representation $\left\langle A, \oplus, *, \sim_{i}\right\rangle$, satisfying (i)-(iv) of Theorem 1.

If $\phi_{1}{ }^{\prime}, \phi_{2}{ }^{\prime}, \phi_{3}{ }^{\prime}$ are any other functions with these properties, then there exist nonzero constants $\alpha_{1}, \alpha_{2}, \alpha_{3}$ such that $\phi_{i}^{\prime}=\alpha_{i} \phi_{i}$, i.e.,

$$
\left(\begin{array}{l}
\phi_{1}{ }^{\prime} \\
\phi_{2}{ }^{\prime} \\
\phi_{3}{ }^{\prime}
\end{array}\right)=\left(\begin{array}{lll}
\alpha_{1} & 0 & 0 \\
0 & \alpha_{2} & 0 \\
0 & 0 & \alpha_{3}
\end{array}\right)\left(\begin{array}{l}
\phi_{1} \\
\phi_{2} \\
\phi_{3}
\end{array}\right) .
$$


Proof. Return to the proof of Theorem 6, in which we have three primaries, $a_{1}, a_{2}, a_{3}$, which are respectively in $A_{2}-A_{1}, A_{1}-A_{2}$, and $A_{1} \cap A_{2}$ (equilibrium red, equilibrium yellow, and achromatic). As before, let $\phi_{1}$ and $\phi_{2}$ be the coefficients of $a_{1}$ and $a_{2}$ in three-primary metameric matching.

To construct $\phi_{3}$, we first observe that for any $c$, there is an equation of form $c \sim_{3} t * a_{3}$. This follows because the structure $\left\langle A, \Theta, *, \sim_{3}\right\rangle$ is proper and 1 -chromatic. So we let $\phi_{3}(c)=t$. Obviously each $\phi_{i}$ provides a representation of $\left\langle A, \oplus, *, \sim_{i}\right\rangle$, and the uniqueness theorem is therefore also immediate, from Theorem 2. It remains only to prove that $\left(\phi_{1}, \phi_{2}, \phi_{3}\right)$ provides a representation of $\langle A, \oplus, *, \sim\rangle$.

Let $\phi_{3}\left(a_{i}\right)=p_{i}$ (note $p_{3}=1$ ). For any $c$ in $A$, we combine Eq. (6) (the trichromatic match for $c$ ) with Axiom $\left(7^{\prime}\right)$ and additivity of $\sim_{3}$ to obtain

$$
\begin{aligned}
{\left[\phi_{3}(c)+\sum_{i=1}^{3} p_{i} s_{i}\right] * a_{3} } & \sim_{3} c \oplus \sum_{i=1}^{3} s_{i} * a_{i} \\
& \sim_{3} \sum_{i=1}^{3} u_{i} * a_{i} \\
& \sim_{3}\left[\sum_{i=1}^{3} p_{i} u_{i}\right] * a_{3} .
\end{aligned}
$$

It follows that $\phi_{3}(c)=\sum_{i=1}^{3} p_{i}\left(u_{i}-s_{i}\right)$. That is, $\phi_{3}$ is a linear combination of the trichromatic matching coefficients relative to $a_{1}, a_{2}, a_{3}$, with coefficients $p_{i}$; since $p_{3} \neq 0, \phi_{3}$ is linearly independent of $\phi_{1}, \phi_{2}$.

Q.E.D.

The above theorem depends crucially on $\sim_{3}$ additivity. There are a variety of techniques for heterochromatic matching, which seem to tap slightly different equivalence relations. Recently, the generalization has emerged that techniques involving minimization of temporal or spatial contrast between two lights yield additivity (see, e.g., Boynton \& Kaiser, 1968) while those that evaluate overall brightness yield nonadditivity (Guth, Donley \& Marrocco, 1969). We may therefore identify $\sim_{3}$ with one of the additive methods. The remaining question is: what sort of theory shall we give for $\sim_{B}$, brightness matching?

The visual significance of the relation $\sim_{B}$ is emphasized by the congruity of results obtained by several quite different methods. Figure 4 compares spectral sensitivity functions obtained from the same subjects and the same viewing conditions by methods of absolute threshold and heterochromatic brightness matching (Wilson, 1964; Jameson, 1969). The two functions coincide. Congruent results were also obtained by magnitude estimation of brightness at different wavelengths.

The problem is akin to that of constructing theories for the nonadditive hue attributes, discussed in the preceding subsection. One may expect a function $f_{B}$, 


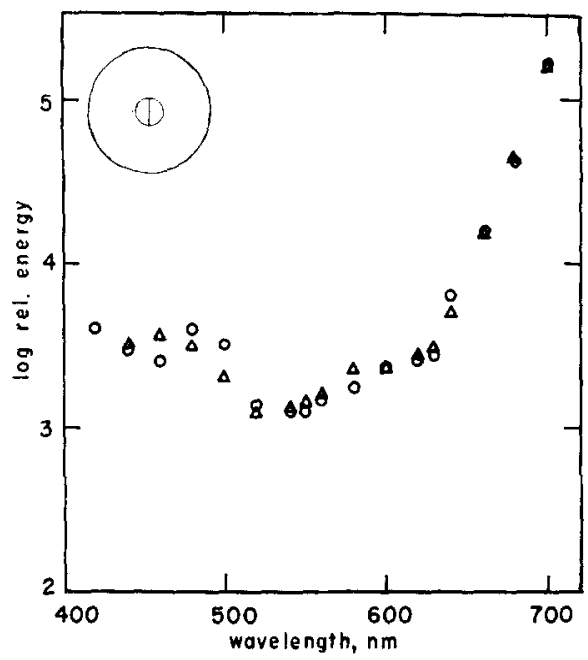

Fig. 4. Comparison of luminous efficiency functions for absolute threshold and for heterochromatic brightness matching (Wilson, 1964). The inset shows the stimulus configuration used to achieve identical viewing conditions. During both threshold determinations and brightness matches, the surround was illuminated with $5500^{\circ} \mathrm{K}$ light and half the bipartite field was illuminated with $550 \mathrm{~nm}$ light, both at $10 \mathrm{~mL}$ luminance. (Reprinted from Jameson, 1969 by permission of Musterschmidt, Göttingen.)

representing quantitative measures of perceived brightness, to depend nonlinearly on all 3 codes that enter Theorem 8 above. The form of the nonlinear function depends on the axioms satisfied by brightness matching. Wilson's results indicate that $f_{B}$ may be an invariant code (see Krantz, 1975.

\section{Hue Matching}

We now turn to the relation $\sim_{H}$, or hue matching, which has been studied primarily in connection with the Bezold-Brücke hue shift and in connection with standardization of the Munsell color notation.

The absence of a Bezold-Brücke shift would mean that hue is invariant with intensity, i.e., $a \sim_{H} t * a$. Such a result would be expected if hue depended only on the ratio $\phi_{1} / \phi_{2}$ of the two chromatic cancellation codes. Since the hue does change, an intuitively attractive idea is to postulate that hue depends rather on the ratio $f_{1} / f_{2}$ of two nonlinear codes that describe perceived intensities of red/green and yellow/blue. This accords both with the intuitive idea that "hue" is the ratio of rcdncss to ycllowness (for orange lights) and with the fact that, as luminance increases, the hue shift consists of yellowness and blueness being emphasized at the expense of redness and greenness. Thus we might expect that $(d / d t) f_{2}(t * a)$ is larger in absolute value than $(d / d t) f_{1}(t * a)$.

Yager and Taylor (1970) obtained $f_{1}$ and $f_{2}$ measures by magnitude estimation of 
yellowness and greenness for yellow and green equilibrium lights varying in intensity. They then tried to predict the hue shift of a yellow-green wavelength, assuming that the shapes of $f_{1}$ and $f_{2}$ as functions of $t$ did not change with wavelength. The results were close, but missed slightly, as one would expect from consideration of veiling. The function $f_{2}$ does change shape when one moves from yellow to yellow/green, because at low luminance the yellow is veiled by greenness.

It seems likely, then, that a suitable theory for $\sim_{H}$ will only be developed in conjunction with a theory of veiling, or a theory of $\sim_{i}^{\prime}$. Quantitative data on both the Bezold-Brücke and the Abney hue shifts are needed; further qualitative generalizations about these phenomena are even more needed.

\section{Saturation}

Saturation actually yields more than a matching relation; lights can be ordered with regard to saturation, with the achromatic lights being least saturated. This relation is of course extremely nonadditive: if $a, b$ are equally saturated complementary lights, then $a \sim_{s} b$ but $a \oplus b \sim_{s} a \oplus a$ is drastically false, since $a \oplus b$ is achromatic.

An intuitive representation for saturation would involve the ratio $\left(\left|f_{1}\right|+\left|f_{2}\right|\right) /\left|f_{3}\right|$ : total absolute values of chromatic codes, relative to achromatic. One might hope that the ordering of such ratios would preserve the ordering $\sim_{s}$ of saturations. The axioms that need to be satisfied for such a representation are in fact well known in the general theory of measurement; the representation is precisely that of distributive conjoint measurement (Krantz \& Tversky, 1971). Testing these axioms presupposes a factorial structure in which the factors are equivalence classes for $\left|f_{1}\right|$-matching, $\left|f_{2}\right|$-matching, and $\left|f_{3}\right|$-matching. Thus, one must do extensive determinations of $\sim_{i}^{\prime}$ and $\sim_{3}$ or $\sim_{B}$ in order to apply the theory here. And we have no guarantee that the intuitive representation will work, nor that the quantitative scales $f_{i}$ constructed by conjoint measurement will give correct quantitative predictions for $\sim_{H}$.

Saturation is also noninvariant with intensity, i.e., $a \sim_{s} t * a$ fails in general. The details of this failure may also provide clues concerning the representation.

\section{The Analogy With Force Measurement}

Since force and color are both represented by 3-dimensional vectors, there is an obvious analogy. In part I of this series I pointed out a less obvious analogy: Not only are the representations isomorphic, but the empirical structures are both Grassmann structures. In the case of color, the elements of the Grassmann structure are functions of wavelength $\lambda$, and in the case of force, the elements are functions of angle $\theta$. The value $a(\lambda)$ is the density of radiant power at wavelength $\lambda$; while $a(\theta)$ is the force-magnitude acting at angle $\theta$. The equivalence relation $\sim$ is metameric matching, in the case of color, and equality of the resultant force, in the case of force. 
A metameric match is determined more or less directly, but equality of resultants is determined by equilibration: $a \sim b$ if and only if an equilibrating element $c$ can be found such that $a \oplus c$ and $b \oplus c$ are both equilibrium configurations.

We are now in a position to recognize that the definition of $\sim$ for force-magnitude configurations is formally identical to the definitions of $\sim_{1}$ and $\sim_{2}$ for opponent-colors theory. In all cases, equivalence is determined by equilibration. More precisely, the theory of the two-dimensional (planar) force table is exactly isomorphic to the theory of 2-chromatic equilibration, relative to the set $B$ of achromatic colors mentioned in the introduction; and the theory of partial equilibria, defined by $A_{1}$ and $A_{2}$, is isomorphic to a theory of partial equilibria of planar forces, relative to any two distinct directions of motion. ${ }^{2}$ The three-dimensional structures for force and color are not isomorphic, however, because the Grassmann structure of lights is proper-in the absence of contrast, there are no lights that are "equilibria" with respect to the third, or whiteblack dimension, and if contrast were used to produce them, such equilibria would not be additive.

\section{Anisotropy of Color Space}

In the case of force equilibria, partial equilibrium can be defined for any direction. Another way of putting this is that the component of the resultant force in any direction is directly observable, by constraining motion to occur in that direction only. But we have developed the color theory in terms of only two special observable "directions" or attributes: red/green and yellow/blue. The question arises, why not define other opponent pairs linearly related to the two just mentioned? Why not a blue-green/orange and a yellow-green/purple dimension?

The reason that these combinations are different can be seen if we consider the definition of partial equilibria and the process of cancellation. What lights are neither blue-green nor orange? Achromatic lights, to be sure, but that is not all. Surely a yellow-green light is neither blue-green nor orange; likewise for a purple light. But the yellow-green "equilibria" are a three-dimensional set, not a two-dimensional one-all mixtures of equilibrium yellow, equilibrium green, and white yield yellowgreens. There is no special distinguished plane, in this set, of colors that are precise equilibria for blue-green/orange. The "equilibrium" set is surely not closed under addition - the sum of a slightly yellowish green and a slightly reddish blue can be very blue-green. Another way to see this is by considering the cancellation process. Suppose that a medium blue-green cancellation standard is used to cancel out orangeness. When will the endpoint be reached? Suppose the orange is a somewhat reddish yellow; then as blue-green is added, the mixture will first become yellow-green, then blue-green. Is the transition from orange to yellow-green the cancellation endpoint, or is it the transition from yellow-green to blue-green? If the former, then the

${ }^{2}$ See also Krantz (1973). 
cancellation coefficient will be proportional to the red/green coefficient; if the latter endpoint is used, the cancellation coefficient will be proportional to the yellow/blue coefficient. So nothing new will be obtained. Moreover, if one tries to cancel the orangeness of a long-wavelength, slightly yellowish light, the transition will not be through yellow-green at all; as blue-green is added, the first transition will be through equilibrium red to purple, then through equilibrium blue to blue green. So now the first transition gives the yellow/blue coefficient, the second one, the red/green coefficient. Our given blue-green standard will only "work" with an orange that is precisely complementary, so that both transitions coincide, via an achromatic equilibrium point.

These considerations indicate that red/green and yellow/blue are special. The system acts as though only two components of the resultant vector are directly observable via partial equilibrium, perhaps because they depend on codes that are really computed in the nervous system. A few othcr codes-whitencss, brightness, hue, saturation-are also directly observable, though they do not involve equilibrium structures. These latter are mainly nonlinear, though whiteness may be linear, as discussed in connection with Theorem 8.

In a way, it is this divergence from the force analogy, via the anisotropy and nonlinearity of the structure of perceptual attributes or codes, that provides the interesting starting points for color theory.

\section{Extensions of Opponent-Colors Theory}

The preceding theory is obviously incomplete, in that the accounts of perceived hue, saturation, and brightness are both tentative and sketchy. The empirical properties of veiling and of various hue shifts particularly need to be determined. It is also incomplete because it does not treat three other broad areas of color data: discrimination, context effects, and defective vision. These were all treated by Hurvich and Jameson in their original development of quantified opponent-colors theory. However, much more empirical work needs to be done before a detailed representation-theorem summary can be expected.

The arca of discrimination is particularly difficult becausc wholly new empirical relations are involved (threshold observations of various sorts) and the proper numerical representation of these, in an opponent-color or any other coordinate system, is uncertain. The present approach may transfer more directly to dealing with context effects and with color defects. In these areas, at least, one can start with the same kind of equilibrium observations as have been discussed here, and one can try to compare the properties of equilibrium sets in altered contexts (adaptation, ${ }^{3}$ contrast) and for various kinds of abnormal observers.

${ }^{3}$ See Cicerone, Krantz, and Larimer (1975). 


\section{REFERENCES}

Boynton, R. M., \& Kaiser, P. K. Vision: The additivity law made to work for heterochromatic photometry with bipartite fields. Science, 1968, 161, 366-368.

Cicerone, C., Krantz, D. H., \& Larimer, J. Opponent-color additivity. III. Effect of moderate chromatic adaptation. Vision Research, 1975,15 (in press).

Guth, S. L., Donley, J., \& Marrocco, R. T. On luminance additivity and related topics. Vision Research, 1969, 9, 537-575.

Hering, E. Zur Lehre vom Lichtsinne. Vienna: C. Gerold's Sohn, 1878.

Hurvich, L. M., \& JAmeson, D. A psychophysical study of white. I. Neutral adaptation. Journal of the Optical Society of America, 1951, 41, 521-527.

HuRvich, L. M., \& JAMESON, D. Some quantitative aspects of an opponent-colors theory. II. Brightness, saturation, and hue in normal and dichromatic vision. Journal of the Optical Society of America, 1955, 45, 602-616.

Indow, T., \& Stevens, S. S. Scaling of saturation and hue. Perception and Psychophysics, 1966, $1,253-271$.

JAMESON, D. Brightness scales and their interpretation. In International colour meeting, Stockholm, 1969, Göttingen: Musterschmidt, 1970. Pp. 377-385.

Jameson, D., \& Hurvich, L. M. Some quantitative aspects of an opponent-colurs theory. I. Chromatic responses and spectral saturation. Journal of the Optical Society of America, 1955, 45, 546-552.

JUDD, D. B. Basic correlates of the visual stimulus. In S. S. Stevens (Ed.), Handbook of experimental psychology. New York: Wiley, 1951.

Krantz, D. H. Fundamental measurement of force and Newton's first and second laws of motion. Philosophy of Science, 1973, 40, 481-495.

KraNTZ, D. H. Color measurement and color theory. I. Representation theorem for Grassmann structures. Journal of Mathematical Psychology, 1975, 12, 283-303.

Krantz, D. H., \& Tversky, A. Conjoint-measurement analysis of composition rules in psychology. Psychological Review, 1971, 78, 151-169.

Larimer, J., Krantz, D. H., \& Cicerone, C. Opponent-color additivity. I. Red/green equilibria. Vision Research, 1974, 14, 1127-1140.

Larimer, J., Krantz, D. H., \& Cicerone, C. Opponent-color additivity. II. Yellow/blue equilibria and nonlinear models. Vision Research, 1975, 15, 723-731.

Priest, I. G., \& Brickwedde, F. G. The minimum perceptible colorimetric purity as a function of dominant wavelength. Journal of the Optical Society of America, 1938, 28, 133-139.

Sinden, R. H. Studies based on the spectral complementaries. Journal of the Optical Society of America, 1923, 7, 1123-1153.

WiLson, B. C. An experimental examination of the spectral luminosity construct. Ph.D. dissertation, New York University, 1964. University Microfilms, Ann Arbor, MI.

YAGER, D., \& TAYLOR, E. Experimental measures and theoretical account of hue scaling as a function of luminance. Perception and Psychophysics, 1970, 5, 360-364.

RECEIVED: November 21, 1974 